\title{
Inhibiting nonmuscle myosin II impedes inflammatory infiltration and ameliorates progressive renal disease
}

\author{
Jin $\mathrm{Si}^{1,2,3}$, Yan $\mathrm{Ge}^{1,3}$, Shougang Zhuang ${ }^{1}$ and Rujun Gong ${ }^{1}$
}

The motor protein nonmuscle myosin II (NMII) through its interaction with the actin cytoskeleton constitutes the machinery of cell crawling and has an important role in driving locomotion and infiltration of immune competent cells during inflammatory response and immune reaction. Blebbistatin is a highly selective inhibitor of NMIl adenosine triphosphatase. This study examined the effect of NMII inhibition by blebbistatin on inflammation. In vitro, blebbistatin markedly induced actinomyosin complex disassembly in various cultured immunocytes, and functionally impaired their motile activity and invasive capacity as assessed by the Boyden chamber motility assay and the matrigel invasion assay. In vivo, in a rat model of acute inflammation induced by tumor necrosis factor, blebbistatin obliterated renal sequestration of circulating fluorescence-labeled macrophages in a dose-dependent fashion. Moreover, in rats with progressive obstructive nephropathy, blebbistatin treatment exhibited a remarkable renoprotective effect, as evidenced by normalized kidney weight, improved gross morphology, and diminished histologic injury in the tubulointerstitium. This beneficial effect was associated with significant amelioration of renal inflammation, consistent with a primary antiinflammatory action by blebbistatin. In addition, in rats with established obstructive nephropathy, blebbistatin pretreated macrophages showed obliterated recruitment into the inflamed renal parenchyma, denoting that blebbistatin directly impedes inflammatory infiltration by immunocytes. Collectively, our findings suggest that inhibition of NMII has a potent and direct anti-inflammatory effect on the basis of impairment of the actinomyosin powered locomotive machinery, which is essential for migration and infiltration of immune competent cells.

Laboratory Investigation (2010) 90, 448-458; doi:10.1038/labinvest.2009.142; published online 11 January 2010

KEYWORDS: cell otility; infiltration; inflammation; kidney; leukocyte; macrophage; nonmuscle myosin II

Inflammation, characterized by the influx of leukocytes or immune competent cells toward the sites of injury or infection, is a protective attempt by the body to remove the injurious stimuli, as well as to initiate the healing process. ${ }^{1-3}$ An inflammatory response of appropriate magnitude and timing is crucial to tissue repair and homeostasis. ${ }^{4}$ Most inflammatory responses are acute and self-limiting; however, an excessive inflammatory reaction may result in critical and fatal conditions, such as systemic inflammatory response syndrome, acute respiratory distress syndrome, and acute renal failure. On the other side, if the inflammatory response is prolonged or frequently relapsing, chronic persistent inflammation develops, in which immune competent cells may promote fibrosis and eventually lead to loss of organ function.,
Central to the pathophysiology of inflammation is tissue infiltration by immune competent cells. ${ }^{3,6}$ Under normal condition, leukocytes continuously patrol the vasculature, alert for signals of inflammation. Proinflammatory substances released by pathogens (eg, lipopolysaccharide) or by damaged tissue (eg, tumor necrosis factor (TNF) activate the leukocytes and initiate the migration of leukocytes to the inflamed area. Leukocyte migration from blood to tissues involves several steps: margination, adhesion to endothelial cells, diapedesis, and chemotaxis. ${ }^{6}$ Cellular motility is indispensable for leukocyte extravasation, migration, and inflammatory infiltration. ${ }^{7,8}$ Cell locomotion is a complex process that requires the coordinated regulation and interaction of numerous reactions. ${ }^{9}$ Nonmuscle myosin II (NMII) is an adenosine triphosphate-driven molecular motor, which

\footnotetext{
'Department of Medicine, Brown University School of Medicine, Providence, RI, USA and ${ }^{2}$ Department of Laboratory Medicine, The Second Affiliated Hospital, Nanjing Medical University, Nanjing, China

Correspondence: Dr R Gong, MD, PhD, Division of Kidney Diseases and Hypertension, Department of Medicine, Rhode Island Hospital, Brown Medical School, 593 Eddy Street, Providence, RI 02903, USA.

E-mail: Rujun_Gong@Brown.edu

${ }^{3}$ These authors contributed equally to this work.

Received 31 July 2009; revised 23 October 2009; accepted 22 November 2009
} 
through the interaction with the actin cytoskeleton forms an indispensable part of the motile machinery for most nonmuscle cells, ${ }^{10}$ including leukocytes. ${ }^{7}$

The (-) enantiomer of blebbistatin [(-)-1-phenyl1,2,3,4-tetrahydro-4-hydroxypyrrolo[2,3-b]-7-methylquinolin-4-one] is a small molecule inhibitor discovered in a screen for inhibitors of NMIIA. ${ }^{11}$ It is a cell-permeable 1-phenyl-12-pyrrolidinone derivative that shows high affinity and selectivity toward NMII adenosine triphosphatase activity with minimal effects on activity of smooth muscle myosin II or other types of myosin. ${ }^{12}$ Blebbistatin was recently found to mitigate the migration of a variety of immune competent cells ${ }^{13-18}$ suggesting that NMII is crucial for immunocyte motility. Consistently, a growing body of evidence reveals that indirect inactivation of NMII through inhibition of myosin light chain kinase ${ }^{19,20}$ or Rho kinase ${ }^{18,21-24}$ impaired leukocyte migration and improved inflammation. Nevertheless, the exact role of NMII in mediating leukocytes' inflammatory response remains uncertain. The purpose of this study was to examine the effect of direct NMII inhibition by blebbistatin on inflammatory infiltration in vitro in cultured immunocytes and in vivo in experimental renal inflammation. We showed that inhibition of NMII by blebbistatin impedes inflammatory infiltration and substantially ameliorates progressive renal disease.

\section{MATERIALS AND METHODS Cell Culture}

The human monocytic leukemia cell line (THP-1), the human T-lymphocyte cell line (Jurkat), and the rat alveolar macrophages (RAMs) were purchased from American Type Culture Collection (ATCC, Manassas, VA, USA). THP-1 and Jurkat cells were cultured in suspension in RPMI supplemented with $10 \%$ fetal bovine serum and RAM cells in Ham's F12K containing $15 \%$ fetal bovine serum. ${ }^{25}$ Cells were maintained in a $37^{\circ} \mathrm{C}, 5 \% \mathrm{CO}_{2}$ incubator and split every 2 days by gentle scraping. Blebbistatin (Sigma, St Louis, MO) was added to the culture at a final concentration of $50 \mu \mathrm{M}$ or otherwise as indicated. Cell viability was assessed by Trypan blue exclusion. At different time points, cells and conditioned media were harvested for further investigation. For fluorescent viable labeling, RAM cells $\left(1 \times 10^{7}\right)$ were incubated in media containing $5 \mathrm{mg} / \mathrm{ml}$ Calcein-AM (Molecular Probes, Eugene, OR) at $37^{\circ} \mathrm{C}$ for $30 \mathrm{~min}$. Excess dye was removed by washing three times with phosphate-buffered saline (PBS). ${ }^{25}$

\section{Western Immunoblot Analysis}

Cultured cells were lysed and animal tissues homogenized in RIPA buffer supplemented with protease inhibitors $(1 \%$ Nonidet P-40, $0.1 \%$ SDS, $100 \mu \mathrm{g} / \mathrm{ml}$ phenylmethysulfonyl fluoride, $0.5 \%$ sodium deoxycholate, $1 \mathrm{mM}$ sodium orthovanadate, $2 \mu \mathrm{g} / \mathrm{ml}$ aprotin, $2 \mu \mathrm{g} / \mathrm{ml}$ leupeptin, $5 \mathrm{mM}$ EDTA in PBS). Protein concentration was determined by using a bicinchoninic acid protein assay kit (Sigma). Samples with equal amounts of total protein $(50 \mu \mathrm{g})$ were fractionated by
$7.5 \sim 15 \%$ SDS-polyacrylamide gels under reducing conditions and analyzed by western immunoblot as previously described. $^{26}$ The antibodies against actin and nonmuscle myosin heavy chain II A (NMHC-IIA) were purchased from Santa Cruz Biotechnology (Santa Cruz, CA) and that for ED-1 from Serotec (Oxford, UK).

\section{Immunoprecipitation}

Immunoprecipitation was carried out using an established method as previously described. ${ }^{27}$ Briefly, cells were washed with ice-cold PBS and then lysed with RIPA buffer supplemented with protease inhibitors. After pre-clearing with normal IgG, cell lysates with equal amount of total protein $\left(0.5 \mathrm{mg}\right.$ of protein) were incubated overnight at $4^{\circ} \mathrm{C}$ with $4 \mu \mathrm{g}$ specific agarose-conjugated antibodies. The precipitated complexes were collected, washed, and separated on SDSpolyacrylamide gels and blotted with various antibodies as indicated.

\section{Boyden Chamber Motility Assay}

RAM cell motility and migration were evaluated using Boyden chamber motogenicity assay with tissue culture-treated Costar transwell filters (Cole-Parmer, Vernon Hills, IL). ${ }^{28}$ RAM cells $\left(5 \times 10^{4}\right)$ were seeded onto the filters $(8-\mu \mathrm{m}$ pore size, $0.33-\mathrm{cm}$ growth area) in the top compartment of the chamber. Vehicle or recombinant monocyte chemotactic protein-1 (MCP-1, R\&D Systems, Minneapolis, MN) was added into the media in the lower chamber at a final concentration of $100 \mathrm{ng} / \mathrm{ml}$. After incubation with or without blebbistatin at $37^{\circ} \mathrm{C}$ for indicated intervals, filters were fixed with 3\% paraformaldehyde in PBS, and stained with $0.1 \%$ Coomassie Blue in 10\% methanol and 10\% acetic acid, and the upper surface of the filters was carefully wiped with a cotton-tipped applicator. Cells that passed through the pores were counted in five non-overlapping $\times 20$ fields and photographed using a Nikon microscope. The experiments were performed in triplicate cultures.

\section{Matrigel Invasion Assay}

Matrigel $\left(1.43 \mathrm{mg} / \mathrm{cm}^{2}\right)$ was added onto the Costar transwell filters of the Boyden chamber to form matrix gels at 1.0-mm depth. RAM cells $\left(5 \times 10^{4}\right)$ in a volume of $100 \mu$ l were added onto the top of the gels. ${ }^{29}$ Vehicle or recombinant rat monocyte chemotactic protein-1 was added into the media in the lower chamber at a final concentration of $100 \mathrm{ng} / \mathrm{ml}$. After incubation with or without blebbistatin at $37^{\circ} \mathrm{C}$ for the indicated intervals, filters were fixed with $3 \%$ paraformaldehyde in PBS, and stained with $0.1 \%$ Coomassie Blue in $10 \%$ methanol and $10 \%$ actic acid, and the upper surface of the filters was carefully wiped with a cotton-tipped applicator. Cells that invaded and migrated across the Matrigel and passed the transwell filter pores toward the lower surface of the filters were counted in five non-overlapping $\times 20$ fields and photographed using a Nikon microscope. The experiments were performed in triplicate cultures. 


\section{Animal Studies}

Male Sprague-Dawley rats with initial weights of 200-250 g were housed in an approved animal care facility and fed standard chow. Experimental protocols were approved by the institution's Animal Care and Use Committee and they conform to USDA regulations and NIH guidelines for humane care and use of laboratory animals. The first study was designed to determine how blebbistatin at different doses affects renal inflammatory infiltration in a model of acute renal inflammation. Briefly, rats were anesthetized and a bolus injection of rat TNF $(2 \mu \mathrm{g} / \mathrm{kg} \mathrm{wt})$ (R\&D Systems) or vehicle was given into the left carotid artery to stimulate the systemic inflammation ${ }^{30}$ and blebbistatin at indicated doses or an equal volume of vehicle was administrated intravenously. Then at different times, fluorescent-labeled RAM cells $\left(1 \times 10^{4}\right)$ resuspended in normal saline were infused through the carotid artery. ${ }^{30}$ For each time point and each blebbistatin dose, five rats were examined. Animals were killed $1 \mathrm{~h}$ after RAM cell infusion and various organs harvested for further investigation. Another study was performed to examine the effect of blebbistatin on experimental progressive kidney injury induced by unilateral ureteral obstruction (UUO). Male Sprague-Dawley rats weighing 200$250 \mathrm{~g}$ were subjected to UUO surgery using an established procedure. ${ }^{31}$ In essence, complete ureteral obstruction was performed under general anesthesia by double-ligating the left ureter using 4-0 silk after a midline abdominal incision. Sham-operated animals had their ureters exposed, manipulated but not ligated. Sham-operated or UUO rats received a daily intravenous injection of blebbistatin or vehicle at a dose of $10 \mathrm{mg} / \mathrm{kg}$ and were killed on day 5 after surgery. For each group and each treatment, five rats were examined. To visualize the direct effect on infiltration of inflammatory cells, another five sham-operated or UUO rats received no treatment after surgery. One hour before being killed on day 5 , they were infused through the carotid artery with fluorescence-labeled RAM cells $\left(1 \times 10^{4}\right)$ that were pretreated with vehicle or blebbistatin $(50 \mu \mathrm{M})$ for $12 \mathrm{~h}$ in the culture. The kidneys were removed and kidney weight assessed. For the excised kidney, one portion was immediately frozen for cryostat sectioning. Another part was fixed in $10 \%$ phosphate-buffered formalin followed by paraffin embedding for histological and immunohistochemical studies. The remaining kidneys were snap-frozen in liquid nitrogen for cryostat sectioning or stored at $-80^{\circ} \mathrm{C}$ for protein extractions. To quantify the fluorescent RAM cells sequestrated in the kidney, kidney homogenates with equal amounts of protein $(100 \mu \mathrm{g})$ were subjected to fluorometric analysis using a Spectramax GEMINI EM fluorescence plate reader (Molecular Devices, Sunnyvale, CA) at an excitation wavelength of $485 \mathrm{~nm}$ and emission at $530 \mathrm{~nm}$.

\section{Morphology and Immunohistochemistry Studies}

Formalin-fixed kidneys were embedded in paraffin and prepared in $3 \mu \mathrm{m}$-thick sections. For general histology, sections were processed for hematoxylin/eosin, periodic acid-Schiff, and Masson-Trichrome staining. Morphology of all sections was assessed by a single observer in a blinded manner. A semiquantitative morphometric score index was used to evaluate the degree of renal tubulointerstitial injury on the basis of the presence of inflammation, basement membrane thickening, dilation, atrophy, sloughing, or interstitial widening as follows: 0 , no changes present; grade 1 , changes present in $<25 \%$ tubulointerstitial area; grade 2, $25 \sim 50 \%$ tubulointerstitial involvement; grade 3, 50 75\% tubulointerstitial involvement; grade $4,75 \sim 100 \%$ tubulointerstitial involvement. A mean score was calculated using the values obtained in 20 random high-power $(\times 400)$ fields in the cortex and medulla per animal in five animals per group. Determination of the degree of interstitial cellularity was carried out on photomicrographs of hematoxylin and eosinstained kidney sections by counting nuclei numbers. Values for individual rats were determined by averaging of 20 randomly chosen high-power fields. Immunoperoxidase staining of rat macrophages (ED-1-positive cells) was performed with a Vectastain $\mathrm{ABC}$ kit (Vector Laboratories, Burlingame, CA). ${ }^{32}$ As a negative control, the primary antibody was replaced by nonimmune serum from the same species. Severity of inflammation was graded by absolute counting of ED-1positive cells in each field and reported as the mean number of cells per square meter in 20 random fields per rat in 5 rats per group. To visualize the fluorescent macrophages sequestered in the tissue, cryostat sections were directly fixed, stained with 4',6-diamidino-2-phenylindole, and then counterstained with Evans blue and mounted with Vectashield mounting medium. ${ }^{30}$ For immunofluorescence microscopy, all sections were stained and analyzed at the same time to exclude artifacts due to variable decay of the fluorochrome. Sections were examined using a Nikon MicrophotFX fluorescence microscope equipped with a Spot II digital camera.

\section{Statistics}

For immunoblot analysis, bands were scanned and the integrated pixel density was determined using a densitometer and the NIH image analysis program. All data are expressed as mean \pm s.d. Statistical analysis of the data from multiple groups was performed by ANOVA followed by StudentNewman-Keuls tests. Data from two groups were compared by Student's $t$-test. $P<0.05$ was considered to be significant.

\section{RESULTS \\ Blebbistatin Induces Actinomyosin Complex Disassembly in Immune Competent Cells}

The actinomyosin complex has an important role in driving locomotion of immune competent cells during inflammatory response and immune reaction. ${ }^{7,10}$ Consistent with previous reports, ${ }^{13,14,17,18} \mathrm{NMII}$ is expressed in all immune competent cells examined, including RAM, THP-1, and Jurkat. MCP-1stimulated macrophages showed an evident physical 
a

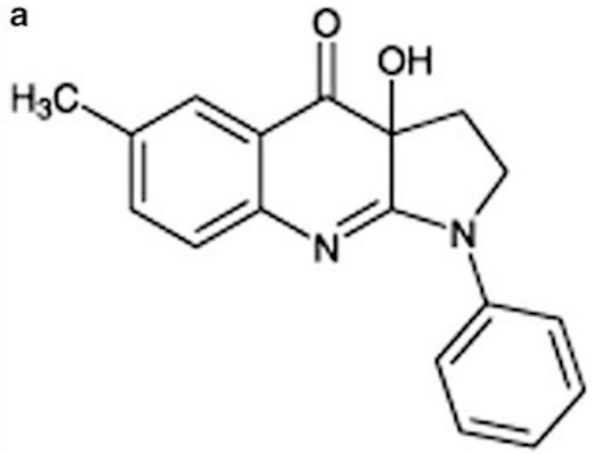

b

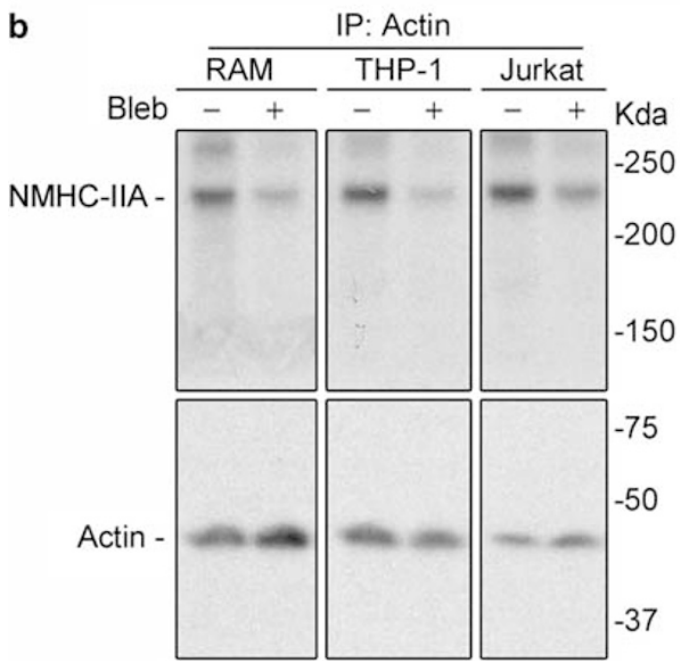

Figure 1 Blebbistatin induces actinomyosin complex disassembly in immune competent cells. (a) The chemical structure of blebbistatin (Bleb), a cell-permeable 1-phenyl-1-2-pyrrolidinone derivative, that shows high affinity and selectivity toward NMII adenosine triphosphatase activity with minimal effects on activity of smooth muscle myosin II or other types of myosin. (b) A variety of immune competent cells, including RAM, THP-1, and Jurkat cells, were stimulated with MCP- $1(100 \mathrm{ng} / \mathrm{ml})$ in the presence or absence of blebbistain $(50 \mu \mathrm{M})$ for $12 \mathrm{~h}$ before the whole cell lysates were collected and subjected to immunoprecipitation. Immunoprecipitates were analyzed for nonmuscle myosin heavy chain IIA (NMHC-IIA) and actin by western immunoblot.

association between actin and myosin (Figure 1b). Blebbistatin, a 1-phenyl-2-pyrrolidinone derivative (Figure 1a), at the concentration of $50 \mu \mathrm{M}$, drastically abrogated the co-precipitation of nonmuscle myosin heavy chain IIA with actin in all cells studied (Figure 1b), consistent with a previous work ${ }^{33}$ showing that inhibition of NMII by blebbistatin induces actinomyosin complex disassembly.

\section{Blebbistatin Retards Motility and Migration of Immune Competent Cells}

To discern the functional consequence of actinomyosin complex disassembly induced by blebbistatin, the cellular motility and migration activity were next assessed using the Boyden chamber motility assay. Shown in Figure 2a, under basal conditions, a few RAM migrated across the pores of the transwell filters (Figure 2a), congruent with a spontaneous motile activity. This was minimally affected by blebbistatinalone treatment (Figure 2b). In contrast, addition of recombinant rat MCP-1 into the media in the lower chamber strikingly induced macrophage migration. Approximately $80 \%$ of the pores in transwell filters were filled with macrophage extensions after $12 \mathrm{~h}$ of MCP-1 treatment (Figure 2c). This effect was substantially diminished in the presence of blebbistatin (Figure 2d). Absolute counting of the migrated cells confirmed that blebbistatin abrogated RAM migration in a dose- (Figure 2e) and time- (Figure 2f) dependent manner.

\section{Invasive Capacity of Immune Competent Cells is Obliterated by Blebbistatin}

The actinomyosin cytoskeleton has also been implicated in cellular invasive activity, ${ }^{15,34}$ which is essential for extravasation and inflammatory infiltration by immune competent cells in the scenario of inflammatory response. We next examined how blebbistatin-induced actinomyosin complex disassembly affects the invasive capacity of macrophages by a matrigel invasion assay. As the cells were seeded on top of matrigel, migration of macrophages across the pores of the transwell filters thus requires both motile activity and invasion of the matrigel. Shown in Figure 3, the addition of MCP-1 in the lower chamber markedly promoted cell invasion into and migration across the matrigel. Approximately $40 \%$ of the pores in transwell filters were filled with macrophage extensions after $12 \mathrm{~h}$ of incubation with MCP-1, as a result of macrophages invading and migrating across the matrigel. This effect was significantly abolished in the presence of blebbistatin. Absolute counting of the migrated cells confirmed that blebbistatin abrogated RAM invasion in a dose- (Figure 3e) and time- (Figure 3f) dependent fashion. Collectively, these findings suggest that blebbistatin hinders the capacity of activated immune competent cells to invade and migrate through the matrigel, which is required by them to disrupt the extracellular matrix and infiltrate toward the inflamed tissues.

\section{Blebbistatin Attenuates Macrophage Sequestration In Vivo in TNF Inflamed Kidney}

To examine the in vivo effect of blebbistatin on immunocyte behavior during inflammatory response and immune reaction, we administrated blebbistatin to a rat model of TNFinduced acute kidney inflammation. One hour before the killing, fluorescent viable RAM were systemically infused into the animals to illustrate the motile activity and invasive capacity of the inflammatory cells. As expected, in normal rat kidney without TNF stimulation, fluorescent RAM was barely observed. Blebbistatin per se made minimal difference at various doses. In contrast, TNF treatment abundantly induced RAM sequestration in the renal parenchyma, representing a typical morphology of acute renal inflammation. Concomitant blebbistatin treatment suppressed 

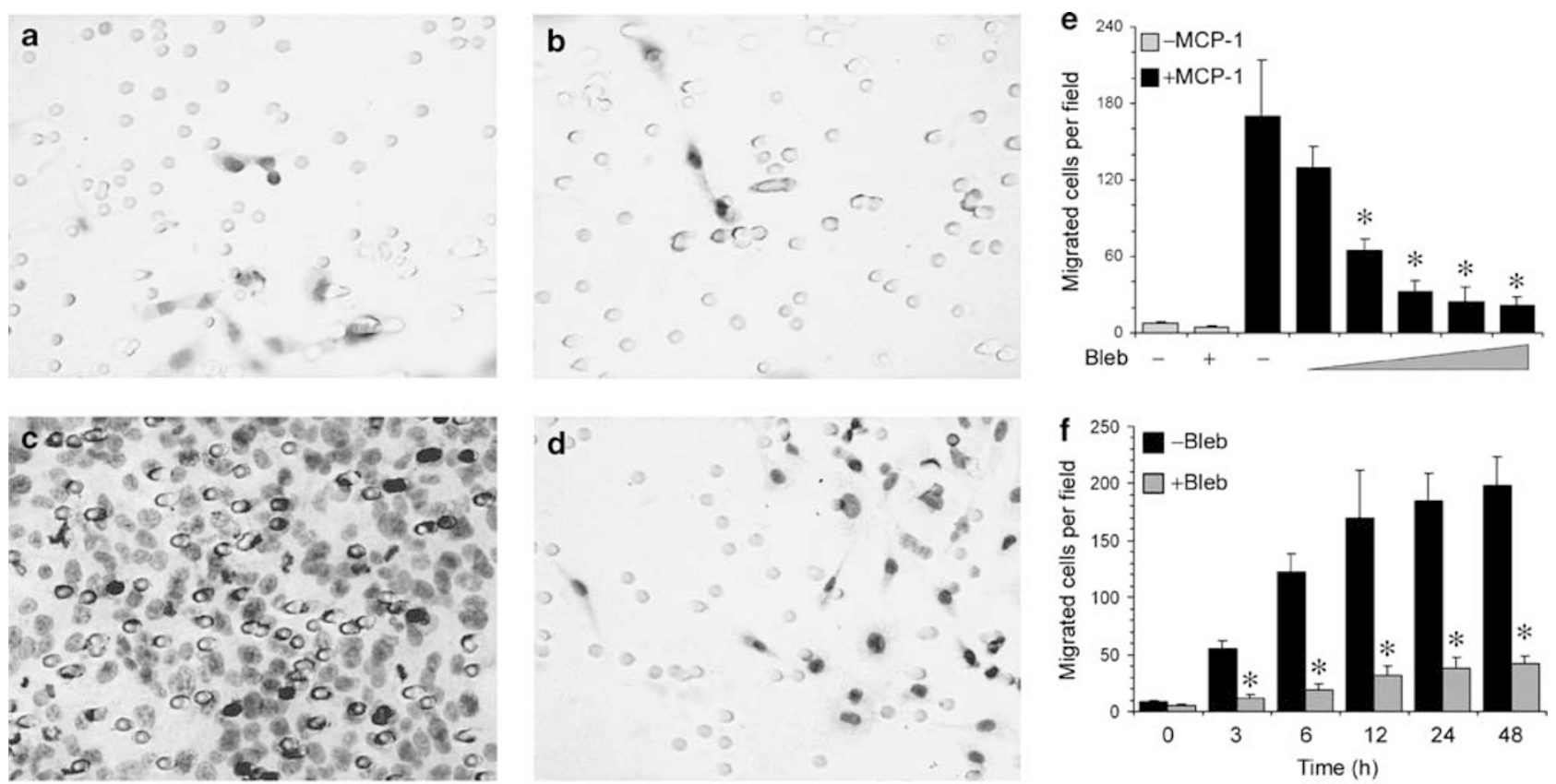

Figure 2 Blebbistatin diminishes macrophage motility and migration as assessed by Boyden chamber motility assay. (a-d) Vehicle (a, b) or recombinant MCP-1 (c, d) was added into the media in the lower chamber. RAM cells were seeded onto the filters in the top compartment of the chamber and treated with $(\mathbf{b}, \mathbf{d})$ or without $(\mathbf{a}, \mathbf{c})$ blebbistatin (Bleb) $(50 \mu \mathrm{M})$ or for $12 \mathrm{~h}$, filters were fixed and cells that passed through the pores were counted. Representative micrographs show RAM that migrated across the pores of the transwell filters after different treatment; (e) Treatment with blebbistatin at $50 \mu \mathrm{M}$ or at varying concentrations $(1,5,10,50,100 \mu \mathrm{M})$ for $12 \mathrm{~h}$ suppresses the RAM migration across the pore; ${ }^{*} P<0.05$ vs MCP-1 alone treated group. (f) Treatment with blebbistatin $(50 \mu \mathrm{M})$ for varying intervals suppresses the RAM migration across the pore; ${ }^{\star} P<0.01$ vs no blebbistatin treatment at the same time points.
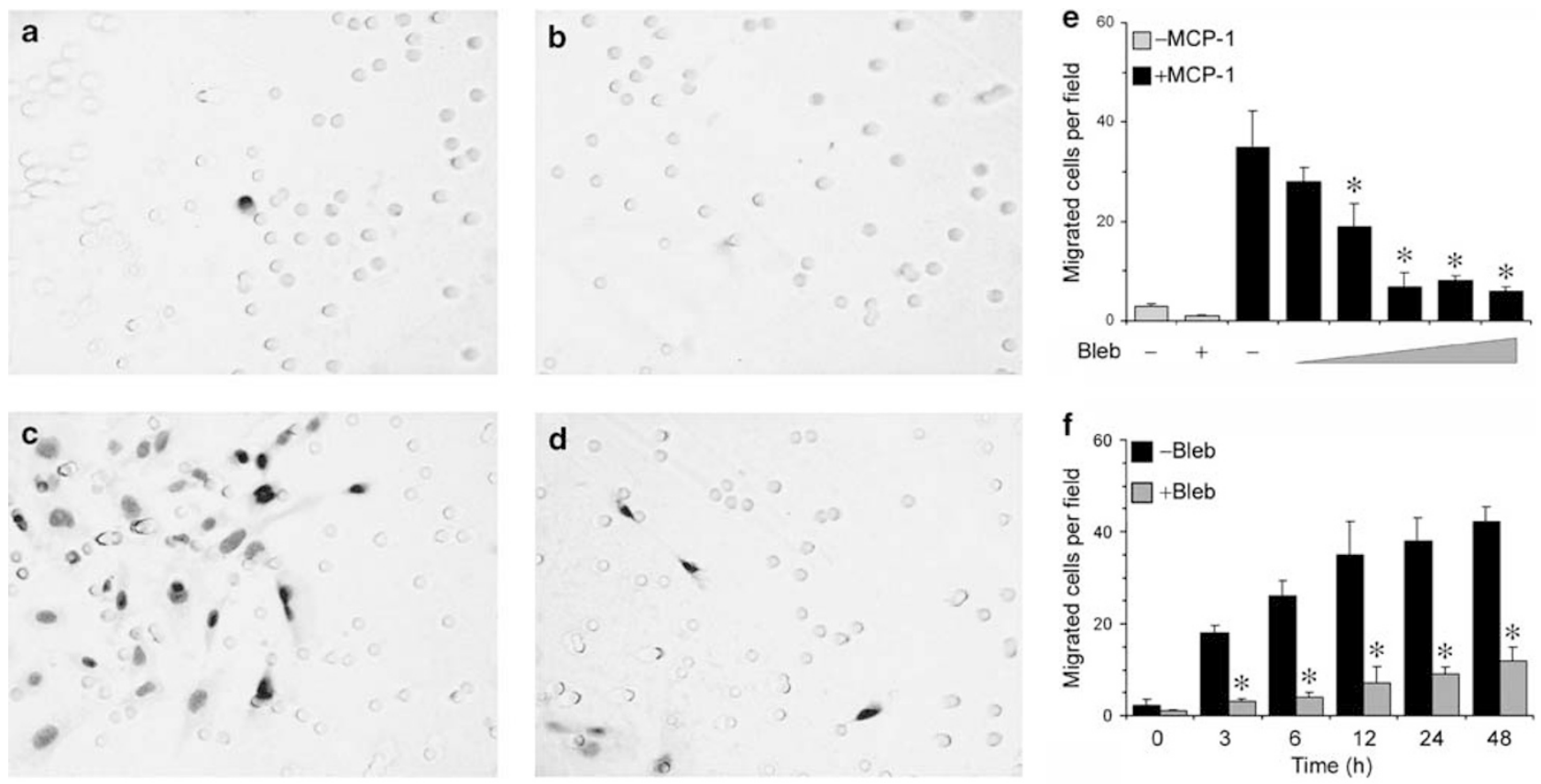

Figure 3 Blebbistatin blunts RAM invasive capacity as assessed by matrigel invasion assay. (a-d) Matrigel was added onto the transwell filters of the Boyden chamber to form matrix layers. Vehicle $(\mathbf{a}, \mathbf{b})$ or recombinant MCP-1 (c, d) was added into the media in the lower chamber. RAM cells were seeded onto the filters in the top compartment of the chamber and treated with (b, d) or without ( $\mathbf{a}, \mathbf{c})$ blebbistatin (Bleb) $(50 \mu \mathrm{M})$ or for $12 \mathrm{~h}$, filters were fixed and cells that invaded and migrated across the Matrigel and passed the transwell filter pores were counted. Representative micropraghs show RAM that migrated across the pores of the transwell filters after different treatment; (e) Treatment with blebbistatin at $50 \mu \mathrm{M}$ or at varying concentrations $(1,5,10,50,100 \mu \mathrm{M})$ for $12 \mathrm{~h}$ suppresses the RAM migration across the pore; ${ }^{\star} P<0.05$ vs MCP-1 alone treated group. (f) Treatment with blebbistatin $(50 \mu \mathrm{M})$ for varying intervals suppresses the RAM migration across the pore; ${ }^{\star} P<0.01$ vs no blebbistatin treatment at the same time points. 

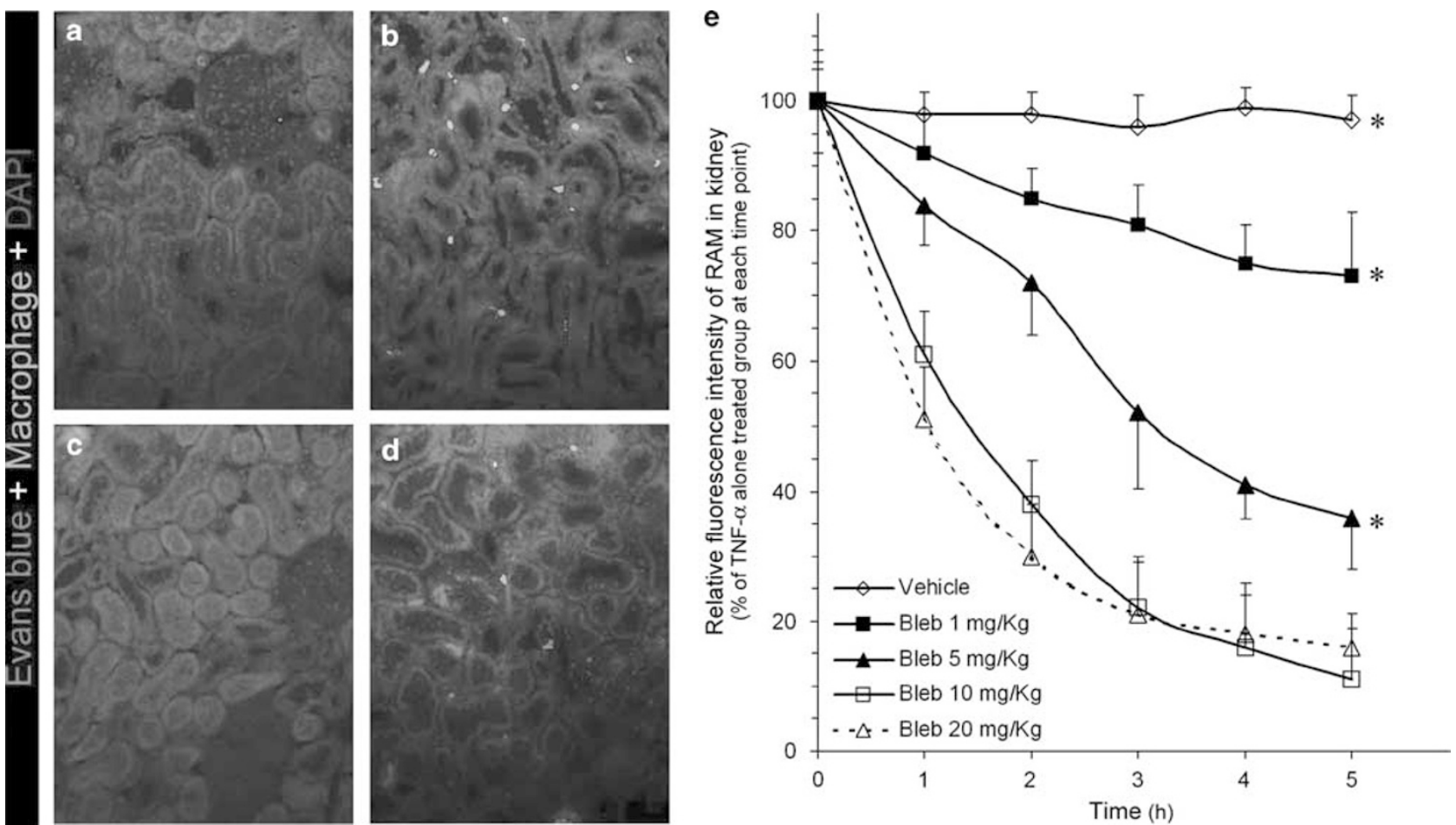

Figure 4 Blebbistatin attenuates macrophage sequestration in vivo in TNF inflamed kidney. Blebbistatin (Bleb) or vehicle was administrated to a rat model of TNF-induced acute kidney inflammation. One hour before killing, fluorescent viable RAM were systemically infused into the animals in order to illustrate the motile activity and invasive capacity of the inflammatory cells. (a-d) Representative micrographs show green fluorescent RAM sequestered in kidneys (counterstained with Evans blue and 4',6-diamidino-2-phenylindole (DAPI)) inflamed with (b, d) or without (a, c) TNF ( $2 \mu \mathrm{g} / \mathrm{kg}$ wt) in the presence (c, d) or absence $(\mathbf{a}, \mathbf{b})$ of blebbistatin $(10 \mathrm{mg} / \mathrm{kg})$. (e) Quantification of RAM cells sequestered in the kidney by fluorometric analysis of kidney homogenates prepared from TNF-inflamed rats treated with blebbistatin at varying doses $(0,1,5,10,20 \mathrm{mg} / \mathrm{kg})$; Data expressed as values from blebbistatin-treated groups relative to those from TNF alone treated group at each time point; ${ }^{\star} P<0.05$ vs other blebbistatin dosages.

TNF-induced RAM sequestration. Quantification of RAM cells sequestered in the kidney by fluorometric analysis of kidney homogenates (Figure 4e) was in agreement with the morphologic findings, and additionally revealed that the inhibitory effect of blebbistatin on RAM infiltration reached a plateau above a dose of $10 \mathrm{mg} / \mathrm{kg}$, suggesting that blebbistatin possesses a dose-dependent anti-inflammatory activity.

\section{Renoprotection by Blebbistatin in Experimental Obstructive Nephropathy}

To further explore the effect of blebbistatin on inflammation in a progressive disease model, we gave sham-operated or UUO rats a daily intravenous injection of blebbistatin and examined the difference in kidney injury. Rats subjected to either sham operation or UUO surgery tolerated well the blebbistatin treatment at a dose of $10 \mathrm{mg} / \mathrm{kg}$ without any discernible manifestation of toxic action or any evidence of adverse effects. Shown in Figure 5a-d, 5 days after complete UUO surgery (Figure 5b), significant distension and dilation of the renal pelvis and calyces were found in the gross morphology of the cross-section of obstructed kidneys, accompanied with a destruction of the kidney parenchymal structure. Blebbistatin had little effect on the gross morphology of the sham-operated kidney (Figure 5c), but markedly restored the gross morphology of the obstructed kidney (Figure 5d). When compared with the sham-operated animals, kidney weight (Figure 5e) increased significantly in UUO rats and this was substantially attenuated by blebbistatin treatment. Microscopic analysis (Figure 6) of the kidney sections indicated that UUO induced a typical type of progressive tubulointerstitial injury, characterized by focal tubular dilation with flattened epithelium, tubular atrophy, epithelial simplification, expansion of the interstitial space, increased extracellular matrix deposition, and proliferation of fibroblast cells and prominent inflammation (Figure $6 c, c^{\prime}$ ). Blebbistatin made little difference to the sham-operated kidneys, which appeared generally normal (Figure 6b, b'), but significantly improved the morphology of the obstructed kidney (Figure 6d, d'). Morphometric analysis showed that blebbistatin greatly deceased the tubulointerstitial injury score (Figure 6e) and diminished the number of infiltrating cells (Figure 6f), denoting a renoprotective action.

\section{Blebbistatin Ameliorates Renal Inflammation in Experimental Obstructive Nephropathy}

Renal inflammation has an important role in kidney destruction and progression of kidney diseases, ${ }^{35,36}$ including obstructive nephropathy. ${ }^{37}$ To explore whether the effect of 

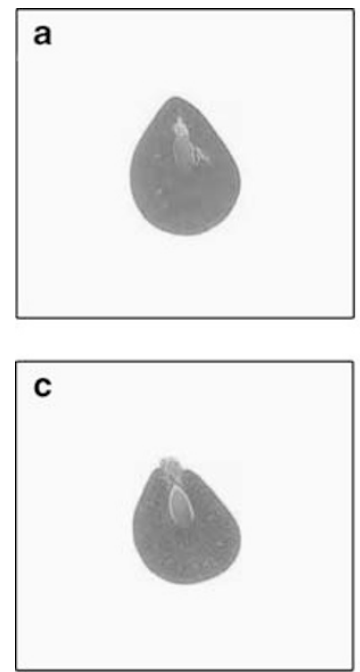
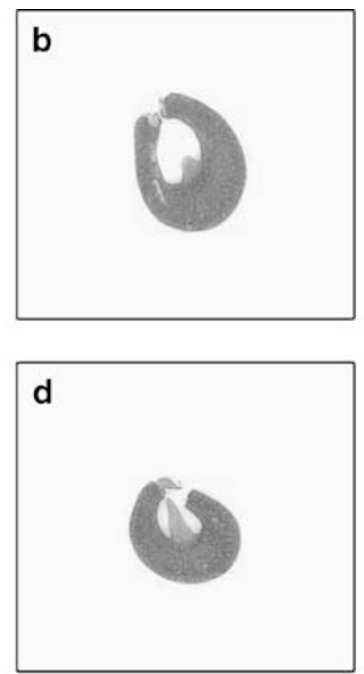

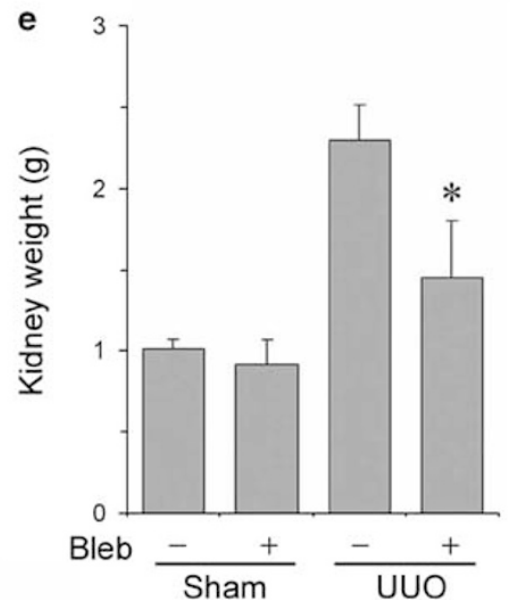

Figure 5 Blebbistatin protects the kidney from UUO-induced injury. (a-d) Representative photos demonstrate the gross morphology of sham operated $(\mathbf{a}, \mathbf{c})$ or UUO (b, d) kidneys from rats treated with vehicle $(\mathbf{a}, \mathbf{b})$ or blebbistatin (Bleb) $(10 \mathrm{mg} / \mathrm{kg})$ (c, d) 5 days after the surgery. (e) Kidney weight was normalized by blebbistatin therapy; ${ }^{*} P<0.05$ vs the UUO group receiving no blebbistatin treatment.
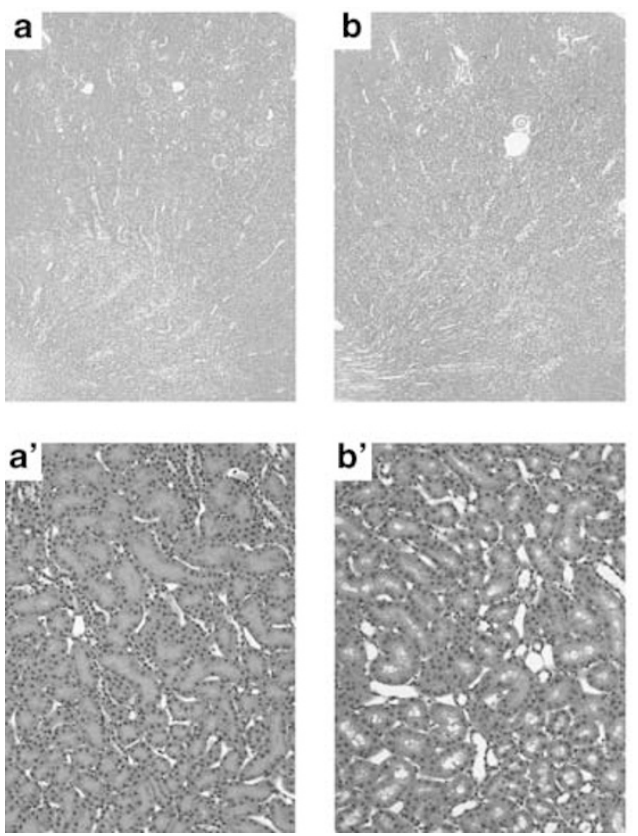
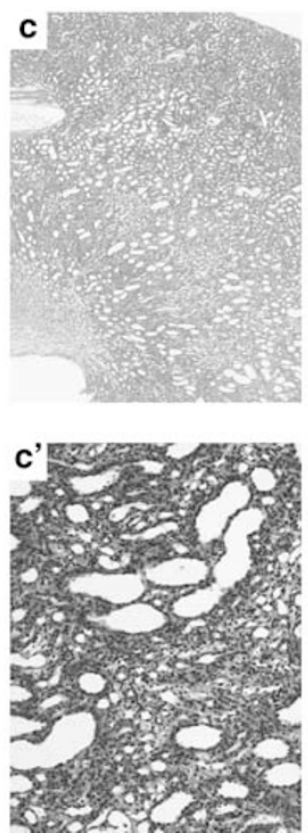
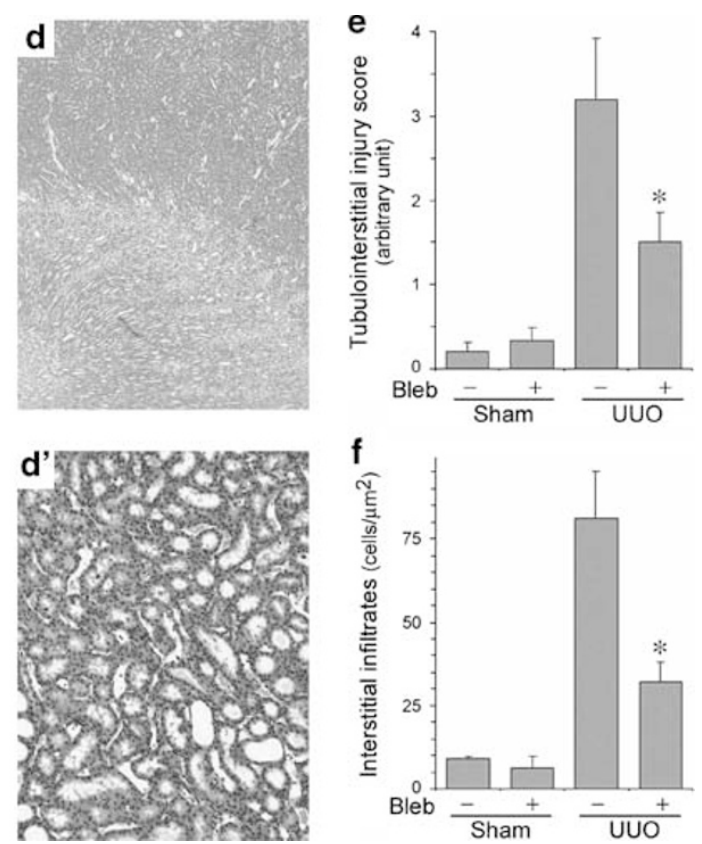

Figure 6 Blebbistatin ameliorates renal histologic injury induced by UUO. (a-d) Representative micrographs show the histology of sham operated (a, b) or UUO (c, d) kidneys from rats treated by vehicle $(\mathbf{a}, \mathbf{c})$ or blebbistatin $(10 \mathrm{mg} / \mathrm{kg})(\mathbf{b}, \mathbf{d}) 5$ days after the surgery, (original magnification, $\times 100)$. $\left(\mathbf{a}^{\prime}-\mathbf{d}^{\prime}\right)$ Magnified images of a-d, respectively (original magnification, $\left.\times 200\right)$; (e) Tubulointerstitial injury scores in the diseased kidneys were improved by blebbistatin therapy; ${ }^{*} P 0.05$ vs the UUO group receiving no blebbistatin treatment. (f) Interstitial infiltrate numbers in the obstructed kidneys were attenuated by blebbistatin; ${ }^{\star} P<0.05$ vs the UUO group receiving no blebbistatin treatment.

blebbistatin on immune competent cells contributes to its renoprotective action, immunohistochemistry staining of ED-1 (Figure 7), a marker for rat macrophage, was carried out on kidney sections. ED- $1^{+}$cells were scantly observed in sham-operated kidneys treated by vehicle or blebbistatin (Figure 7c). Tubulointerstitial infiltration of ED-1-positive cells was abundantly induced 5 days after ureteral obstruction (Figure 7b, b') but was strikingly abrogated by blebbistatin treatment (Figure $7 \mathrm{~d}, \mathrm{~d}$ '). The morphologic findings were corroborated by immunoblot analysis (Figure 7e) of kidney homogenates for the ED-1 molecule and then further revalidated by absolute counting (Figure 7f) of ED-1-positive 

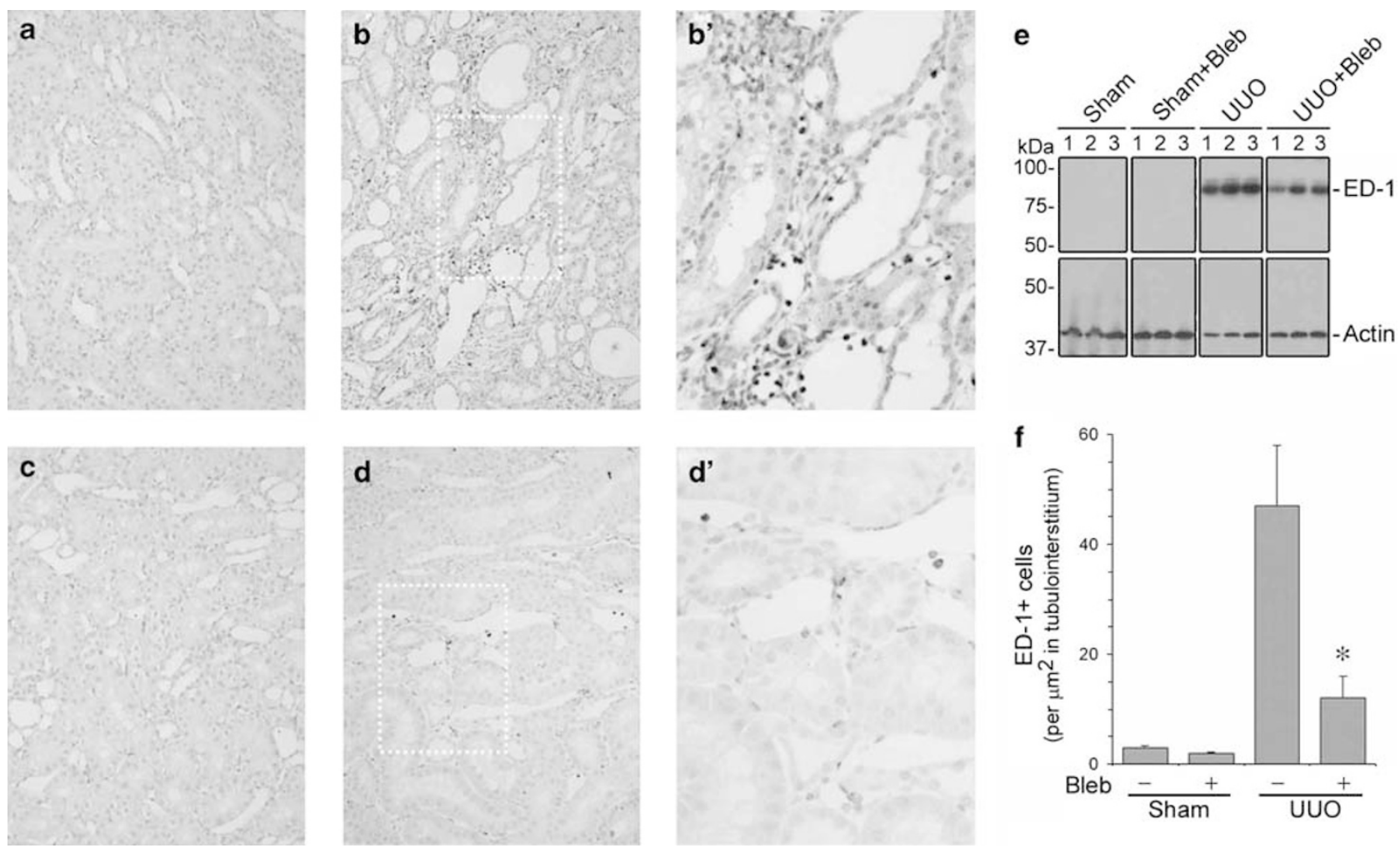

Figure 7 Blebbistatin treatment markedly alleviated renal inflammation and reduced macrophage infiltration in the obstructed kidney. (a-d) Representative micrographs show the immunohistochemistry staining of rat macrophages (ED-1 positive cells) in sham operated (a, $\mathbf{c})$ or UUO (b, d) kidneys from rats treated by vehicle $(\mathbf{a}, \mathbf{b})$ or blebbistatin $(10 \mathrm{mg} / \mathrm{kg})(\mathbf{c}, \mathbf{d}) 5$ days after the surgery; original magnification, $\times 200$. (b', $\left.\mathbf{d}^{\prime}\right)$ Magnified images of $\mathbf{b}$ and $\mathbf{d}$ respectively; original magnification, $\times 400$. (e) Immunoblot analysis on kidney homogenates for ED-1 and actin; (f) Absolute counting of ED-1 positive cells in tubulointerstitium in kidney sections after immunohistochemistry staining as shown in $a-d ;{ }^{\star} P<0.05$ vs the UUO group receiving no blebbistatin treatment.

cells in the sections, all supporting that blebbistatin ameliorates renal inflammation in obstructed nephropathy.

\section{Blebbistatin Directly Impedes Inflammatory Infiltration into the Diseased Kidney}

To determine whether blebbistatin-induced improvement of renal inflammation in the obstructed kidney is ascribed to its direct effect on inflammatory cell infiltration, rats were allowed to develop kidney injury without any treatment for 5 days after the surgery. Then, fluorescence-labeled RAM cells pretreated with blebbistatin or vehicle were infused systemically and animals killed in $1 \mathrm{~h}$. We assumed that through this way the difference in the sequestration of RAM cells in the obstructed kidney would be largely attributable to the direct effect of blebbistatin on RAM cells. Shown in Figure 8, vehicle- or blebbistatin-pretreated RAM showed minimal infiltration in the sham-operated kidney (Figure 8a, c). However, in the obstructed kidneys, abundant vehicle-pretreated RAM cells (Figure 8b, b') but significantly fewer blebbistatin-treated RAM cells (Figure 8d, d') were found sequestered, although the kidneys had an equal magnitude of histologic injury, suggesting that blebbistatin treatment directly hindered RAM migration and infiltration into the diseased kidney. Again, quantification of RAM cells sequestered in the kidney by fluorometric analysis of kidney homogenates (Figure 8e) or by absolute counting of fluorescent cells in each kidney section (Figure 8f) corroborated the morphologic findings.

\section{DISCUSSION}

Regardless of the original etiology, inflammation is an important final common pathway for most progressive diseases. ${ }^{38-40}$ Inflammation, marked by tissue infiltration by excessive immune competent cells, induces tissue fibrogenesis and eventually drives progression to end-stage organ failure. ${ }^{41,42}$ Epidemiologic data suggest that inflammation is one of the potentially modifiable risk factors in most forms of chronic diseases, including cardiovascular diseases, ${ }^{43}$ diabetes, ${ }^{44}$ chronic obstructive pulmonary diseases, ${ }^{45}$ and chronic kidney diseases. ${ }^{46}$ The current therapy of inflammation is mainly limited to nonsteroidal anti-inflammatory drugs and immunosuppressants, which are widely used in patients with excessive or chronic inflammation. ${ }^{4}$ These treatments suppress the production of inflammatory mediators by injured tissues and modulate the function of inflammatory cells. However, no currently 

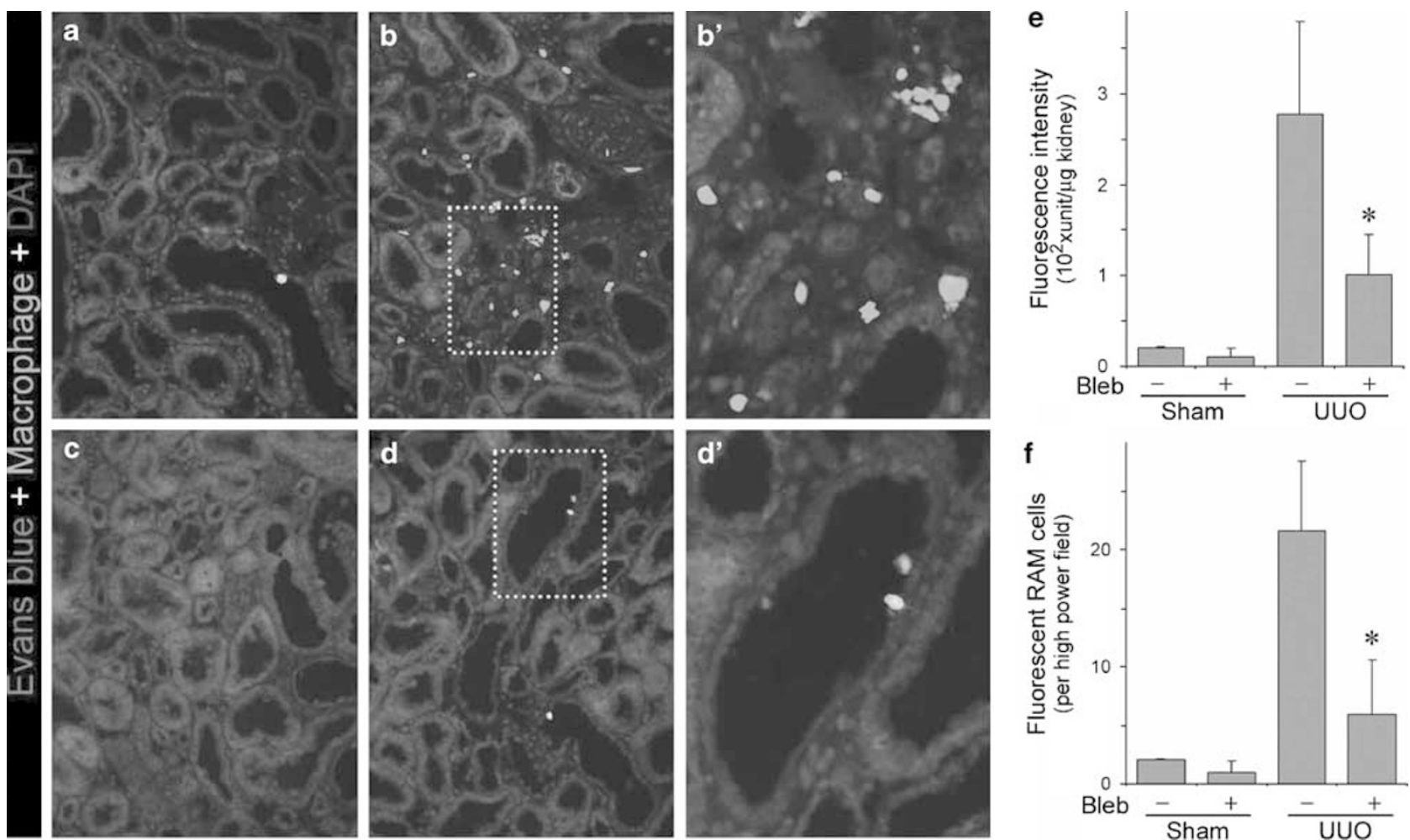

Figure 8 Blebbistatin pretreated macrophage demonstrated reduced recruitment into the diseased kidney. After the UUO surgery or sham operation, rats were allowed to develop kidney injury without any treatment for 5 days. Then, RAM cells pretreated with blebbistatin or vehicle for $12 \mathrm{~h}$ were labeled with fluorescence and infused systemically and animals sacrificed in one hour. (a-d) Representative micrographs show green fluorescent RAM sequestered in sham operated $(\mathbf{a}, \mathbf{c})$ or UUO (b, d) kidneys [counterstained with Evans blue and 4',6-diamidino-2-phenylindole (DAPI)] from rats infused with vehicle (a, b) or blebbistatin $(50 \mu \mathrm{M})$ pretreated $(\mathbf{c}, \mathbf{d})$ RAM; original magnification, $\times 200$. ( $\mathbf{b}^{\prime}$, d') Magnified images of $\mathrm{b}$ and d, respectively; original magnification, $\times 400$. (f) Quantification of RAM cells sequestered in the kidney by fluorometric analysis of kidney homogenates prepared from each group; ${ }^{\star} P<0.05$ vs the UUO group receiving no blebbistatin treatment. (f) Absolute counting of fluorescent RAM cells in kidney sections as shown in a-d; ${ }^{\star} P<0.05$ vs the UUO group receiving no blebbistatin treatment.

available treatment is completely effective, and a considerable number of patients suffer from the side effects of these treatments. Mechanistically, these treatments neither directly target the locomotion of inflammatory cells nor effectively arrest the inflammatory infiltration by leukocytes. To the best of our knowledge, this study is the first to show that inhibiting leukocyte motility impedes inflammatory infiltration and ameliorates progressive kidney disease.

The molecular mechanisms that drive leukocyte migration and infiltration are still not fully understood. Recent studies suggest that the locomotive machinery has a pivotal role. ${ }^{7,8}$ This machinery of cell crawling is composed of a wellorchestrated molecular network including cytoskeleton actin and motor proteins such as NMII in nonmuscle cells, which on activation form the actinomyosin apparatus and mediates leukocyte migration. ${ }^{9,10}$ Evidence suggests that the activity of NMII is essential for leukocyte motility. In a study by Eddy et $a l,{ }^{16}$ inhibition of NMII activity indirectly by myosin light chain kinase inhibitors or through buffering of intracellular $\mathrm{Ca}^{2+}$ transients retarded neutrophil motility because of impairment of uropod retraction and detachment. This effect resembled that of direct NMII suppression by 2,3-butane- dione monoxime, a myosin blockade, ${ }^{47}$ although the specificity of this chemical inhibitor is not as satisfactory as newly discovered ones such as blebbistatin. ${ }^{12}$ Consistent with these findings, NMII was lately found to be associated with the uropod during T-lymphocyte crawling ${ }^{13,14}$ and knockdown of NMIIA by RNA interference resulted in a defect in tail retraction in $\mathrm{T}$ cells and reduced its crawling activity on either ICAM-1 coated $^{13}$ or plain culture surface. ${ }^{14}$ Besides neutrophils and T lymphocytes, NMII is also indispensable for migration and chemotaxis in macrophages and other leukocytes. ${ }^{17,18}$ Moreover, NMII is also required for mammalian epithelial morphogenesis and cell invasion..$^{15,29,34}$ For instance, in epithelial cells grown in Matrigel matrix, inhibition of NMII triggered the development of membrane extensions and regulated alterations of cell shape, which are both necessary for initial transformation between the cyst and the tubule and later for tubule branching. ${ }^{29}$ In addition, in cultured pancreatic adenocarcinoma cells, inhibition of NMII activity blunted cellular migration and invasiveness. ${ }^{34}$ This effect was not likely to be attributable to the enhanced extracellular matrix proteolysis, but to the mechanical effect on cell migration, because the activity of matrix metallo- 
proteinases was unaffected; however, cell spreading on its substrate was markedly impaired after NMII inhibition. ${ }^{34}$ Consistently, in our study, selective inhibition of NMII adenosine triphosphate induced actinomyosin complex disassembly and diminished macrophage motility and invasive capacity. In addition to the role in leukocyte migration, NMII has been implicated in innate immune function. In support of this, NMII inhibitors or specific knockdown of the myosin IIA heavy chain by RNA interference impaired cytotoxicity, membrane fusion of lytic granules, and granzyme secretion in natural killer cells. ${ }^{48}$ Thus, NMII is required for cytolytic granule exocytosis in innate immune response. Moreover, accumulating evidence suggests that NMII might also have an important role in acquired immune recognition, such as B cell receptor-driven antigen processing and presentation. In a recent study by Vascotto et al, ${ }^{49}$ NMII was found to be activated on B-cell receptor engagement and to associate with MHC class II-invariant chain complexes. NMII inhibition or depletion compromised the convergence and concentration of MHC class II and B-cell receptor-antigen complexes into lysosomes devoted to antigen processing. Accordingly, the formation of MHC class II-peptides and subsequent CD4 T-cell activation were impaired in cells lacking myosin II activity. Collectively, more and more data suggest that NMII is an essential element mediating motility, invasiveness, and function of immunocytes during inflammatory response and thus might serve as a potential therapeutic target for treating inflammatory infiltration.

Blebbistatin, a 1-phenyl-2-pyrrolidinone derivative, has been shown to selectively inhibit NMII, but does not inhibit myosin from classes I, V, and X. It behaves as an uncompetitive inhibitor and functions by binding the large cleft in the motor domain, which opens and closes during the contractile cycle. ${ }^{11,12}$ Blebbistatin specifically stabilizes the metastable or transition state of myosin. ${ }^{12}$ Binding of blebbistatin to myosin II leads to a long-lived complex of myosin with adenosine diphosphate and inorganic phosphate, which occurs before the force-generating step catalyzed by the release of phosphate on the rebinding of myosin with actin. ${ }^{50}$ Thus, blebbistatin inhibits the transition into force-producing states. ${ }^{12}$ Blebbistatin has been widely accepted and used as a highly selective blockade for NMII. Previous studies have shown that blebbistatin was able to paralyze the myosin power stroke, inhibit motility, and blunt migration and chemotaxis in a variety of cultured leukocytes, ${ }^{13,14,16,18,19}$ suggesting that inhibition of NMII might ameliorate inflammatory infiltration. Indeed, evidence suggests that inhibition of Rho kinase, a regulator myosin phosporylation, suppressed myosin activity and disrupted macrophage migration. ${ }^{18,21}$ In vivo, long-term treatment with Rho kinase inhibitor attenuated coronary vascular inflammation and induced a regression of atherosclerotic coronary lesions, ${ }^{23,24}$ possibly by preventing migration of macrophages. ${ }^{18,24}$ Nevertheless, the in vivo effect of direct NMII inhibition by blebbistatin has barely been investigated. In this study, blebbistatin was administrated for the first time to rats with progressive kidney disease. The optimal dose at which blebbistatin could effectively ameliorate inflammation in vivo has barely been studied. To this end, a rat model of TNF-induced acute kidney inflammation was treated with blebbistatin at various doses to determine the effective dose. Congruent with the observation in cultured macrophages, RAM sequestration to the inflamed kidney was obliterated by blebbistatin in a dose-dependent manner. However, blebbistatin at doses higher than $10 \mathrm{mg} / \mathrm{kg}$ did not exhibit further inhibitory effect, suggesting that inhibition of RAM infiltration by blebbistatin reached a plateau above a dose of $10 \mathrm{mg} / \mathrm{kg}$. Therefore, the dose of blebbistatin at $10 \mathrm{mg} / \mathrm{kg}$ was chosen for the following animal studies. Animals tolerated well the blebbistatin treatment without any discernible manifestation of toxic action or any evidence of side effects. Consistent with the in vitro findings, infiltration of immune competent cells in the inflamed kidney was substantially attenuated by blebbistatin and the gross kidney injury improved, suggesting that blebbistatin might be a promising treatment for inflammation. One limitation of this study is that UUO is an acute model and is unlike most human forms of progressive kidney disease. In addition, the pathogenic mechanisms of the UUO model may not be applicable to the majority of human kidney disease. Thus, in-depth studies are merited to validate the effect of blebbistatin in other types of progressive kidney injury.

In summary, we have found that inhibition of NMII by blebbistatin, a small molecule inhibitor of NMII adenosine triphosphatase, disrupted the locomotive machinery of the actinomyosin system and impaired cellular motility and invasiveness in immune competent cells. In addition, in a rat model of progressive kidney disease induced by unilateral ureteral obstruction, treatment with blebbistatin successfully arrested macrophage infiltration to the renal parenchyma, ameliorated inflammation, and improved renal injury. Our findings suggest that inhibition of NMII in vivo is possible with selective small molecule inhibitors, such as blebbistatin, and might represent a new therapeutic strategy to treat human inflammatory diseases.

\section{ACKNOWLEDGEMENTS}

This study was supported by the Rhode Island Medical Foundation, the Lifespan Developmental Grant, and the Young Investigator Research Award from the Foundation for Health (to RG). We are grateful to Ms Tolbert for the technical assistance in establishing the animal models in the pilot study.

\section{DISCLOSURE/CONFLICT OF INTEREST}

The authors declare no conflict of interest.

1. Levy JH. The human inflammatory response. J Cardiovasc Pharmacol 1996;27(Suppl 1):S31-S37.

2. Oberyszyn TM. Inflammation and wound healing. Front Biosci 2007;12:2993-2999.

3. Ward PA, Marks RM. The acute inflammatory reaction. Curr Opin Immunol 1989;2:5-9.

4. Nathan C. Points of control in inflammation. Nature 2002;420:846-852. 
5. Gong R. Multi-target anti-inflammatory action of hepatocyte growth factor. Curr Opin Investig Drugs 2008;9:1163-1170.

6. Ali H, Haribabu B, Richardson RM, et al. Mechanisms of inflammation and leukocyte activation. Med Clin North Am 1997;81:1-28.

7. Vicente-Manzanares M, Sancho D, Yanez-Mo M, et al. The leukocyte cytoskeleton in cell migration and immune interactions. Int Rev Cytol 2002;216:233-289.

8. Norberg B, Bandmann U, Rydgren L. Amoeboid movement in human leucocytes: basic mechanisms, cytobiological and clinical significance. J Mechanochem Cell Motil 1977;4:37-53.

9. Stupack DG, Cho SY, Klemke RL. Molecular signaling mechanisms of cell migration and invasion. Immunol Res 2000;21:83-88.

10. Jay PY, Pham PA, Wong SA, et al. A mechanical function of myosin II in cell motility. J Cell Sci 1995;108(Part 1):387-393.

11. Limouze J, Straight AF, Mitchison T, et al. Specificity of blebbistatin, an inhibitor of myosin II. J Muscle Res Cell Motil 2004;25:337-341.

12. Allingham JS, Smith R, Rayment I. The structural basis of blebbistatin inhibition and specificity for myosin II. Nat Struct Mol Biol 2005;12: 378-379.

13. Morin NA, Oakes PW, Hyun YM, et al. Nonmuscle myosin heavy chain IIA mediates integrin LFA-1 de-adhesion during $T$ lymphocyte migration. J Exp Med 2008;205:195-205.

14. Jacobelli J, Chmura SA, Buxton DB, et al. A single class II myosin modulates $T$ cell motility and stopping, but not synapse formation. Nat Immunol 2004;5:531-538.

15. Bastian $\mathrm{P}$, Lang $\mathrm{K}$, Niggemann $\mathrm{B}$, et al. Myosin regulation in the migration of tumor cells and leukocytes within a three-dimensional collagen matrix. Cell Mol Life Sci 2005;62:65-76.

16. Eddy RJ, Pierini LM, Matsumura F, et al. $\mathrm{Ca}^{2+}$-dependent myosin II activation is required for uropod retraction during neutrophil migration. J Cell Sci 2000;113(Part 7):1287-1298.

17. Lammermann T, Bader BL, Monkley SJ, et al. Rapid leukocyte migration by integrin-independent flowing and squeezing. Nature 2008;453: 51-55.

18. Nakayama M, Amano M, Katsumi A, et al. Rho-kinase and myosin II activities are required for cell type and environment specific migration. Genes Cells 2005;10:107-117.

19. Xu J, Gao XP, Ramchandran R, et al. Nonmuscle myosin light-chain kinase mediates neutrophil transmigration in sepsis-induced lung inflammation by activating beta2 integrins. Nat Immunol 2008;9: 880-886.

20. Simoes RL, Fierro IM. Involvement of the Rho-kinase/myosin light chain kinase pathway on human monocyte chemotaxis induced by ATL-1, an aspirin-triggered lipoxin A4 synthetic analog. J Immunol 2005; 175:1843-1850.

21. Doe C, Bentley R, Behm DJ, et al. Novel Rho kinase inhibitors with antiinflammatory and vasodilatory activities. J Pharmacol Exp Ther 2007;320:89-98.

22. Kureishi $Y$, Kobayashi S, Amano M, et al. Rho-associated kinase directly induces smooth muscle contraction through myosin light chain phosphorylation. J Biol Chem 1997;272:12257-12260.

23. Mallat Z, Gojova A, Sauzeau V, et al. Rho-associated protein kinase contributes to early atherosclerotic lesion formation in mice. Circ Res 2003;93:884-888.

24. Miyata $\mathrm{K}$, Shimokawa $\mathrm{H}$, Kandabashi $\mathrm{T}$, et al. Rho-kinase is involved in macrophage-mediated formation of coronary vascular lesions in pigs in vivo. Arterioscler Thromb Vasc Biol 2000;20:2351-2358.

25. Gong R, Rifai A, Dworkin LD. Anti-inflammatory effect of hepatocyte growth factor in chronic kidney disease: targeting the inflamed vascular endothelium. J Am Soc Nephrol 2006;17:2464-2473.

26. Gong R, Liu Z, Li L. Epistatic effect of plasminogen activator inhibitor 1 and $\beta$-fibrinogen genes on risk of glomerular microthrombosis in lupus nephritis: interaction with environmental/clinical factors. Arthritis Rheum 2007;57:1608-1617.
27. Gong R, Rifai A, Ge Y, et al. Hepatocyte growth factor suppresses proinflammatory NFkappaB activation through GSK3beta inactivation in renal tubular epithelial cells. J Biol Chem 2008;283:7401-7410.

28. Pixley FJ, Xiong Y, Yu RY, et al. BCL6 suppresses RhoA activity to alter macrophage morphology and motility. J Cell Sci 2005;118: 1873-1883.

29. Ivanov Al, Hopkins AM, Brown GT, et al. Myosin II regulates the shape of three-dimensional intestinal epithelial cysts. J Cell Sci 2008;121:1803-1814.

30. Gong R, Rifai A, Dworkin LD. Hepatocyte growth factor suppresses acute renal inflammation by inhibition of endothelial E-selectin. Kidney Int 2006:69:1166-1174.

31. Kawai T, Masaki T, Doi S, et al. PPAR-gamma agonist attenuates renal interstitial fibrosis and inflammation through reduction of TGF-beta. Lab Invest 2009;89:47-58.

32. Chen S, Ge Y, Si J, et al. Candesartan suppresses chronic renal inflammation by a novel antioxidant action independent of AT1R blockade. Kidney Int 2008;74:1128-1138.

33. Wang HH, Tanaka H, Qin X, et al. Blebbistatin inhibits the chemotaxis of vascular smooth muscle cells by disrupting the myosin II-actin interaction. Am J Physiol Heart Circ Physiol 2008;294:H2060-H2068.

34. Duxbury MS, Ashley SW, Whang EE. Inhibition of pancreatic adenocarcinoma cellular invasiveness by blebbistatin: a novel myosin II inhibitor. Biochem Biophys Res Commun 2004;313:992-997.

35. Lan HY, Nikolic-Paterson DJ, Mu W, et al. Local macrophage proliferation in progressive renal injury. Contrib Nephrol 1996;118:100-108.

36. Rodriguez-Iturbe $\mathrm{B}$, Pons $\mathrm{H}$, Herrera-Acosta J, et al. Role of immunocompetent cells in nonimmune renal diseases. Kidney Int 2001;59:1626-1640.

37. Chevalier RL. Pathogenesis of renal injury in obstructive uropathy. Curr Opin Pediatr 2006;18:153-160.

38. Eddy AA. Molecular basis of renal fibrosis. Pediatr Nephro 2000;15:290-301.

39. Eddy AA, Neilson EG. Chronic kidney disease progression. J Am Soc Nephrol 2006;17:2964-2966.

40. Remuzzi G, Bertani T. Pathophysiology of progressive nephropathies. N Engl J Med 1998;339:1448-1456.

41. Chatziantoniou C, Dussaule JC. Insights into the mechanisms of renal fibrosis: is it possible to achieve regression? Am J Physiol Renal Physiol 2005;289:F227-F234.

42. Martin P, Leibovich SJ. Inflammatory cells during wound repair: the good, the bad and the ugly. Trends Cell Biol 2005;15:599-607.

43. Ridker PM, Cushman M, Stampfer MJ, et al. Inflammation, aspirin, and the risk of cardiovascular disease in apparently healthy men. $\mathrm{N}$ Engl J Med 1997;336:973-979.

44. Theuma $P$, Fonseca VA. Inflammation and emerging risk factors in diabetes mellitus and atherosclerosis. Curr Diab Rep 2003;3:248-254.

45. Groenewegen KH, Postma DS, Hop WC, et al. Increased systemic inflammation is a risk factor for COPD exacerbations. Chest 2008;133:350-357.

46. Haroun MK, Jaar BG, Hoffman SC, et al. Risk factors for chronic kidney disease: a prospective study of 23534 men and women in Washington County, Maryland. J Am Soc Nephrol 2003;14:2934-2941.

47. Ostap EM. 2,3-Butanedione monoxime (BDM) as a myosin inhibitor. J Muscle Res Cell Motil 2002;23:305-308.

48. Andzelm MM, Chen X, Krzewski $K$, et al. Myosin IIA is required for cytolytic granule exocytosis in human NK cells. J Exp Med 2007;204:2285-2291.

49. Vascotto F, Lankar D, Faure-Andre G, et al. The actin-based motor protein myosin II regulates $\mathrm{MHC}$ class II trafficking and BCR-driven antigen presentation. J Cell Biol 2007;176:1007-1019.

50. Kovacs $M$, Toth J, Hetenyi $C$, et al. Mechanism of blebbistatin inhibition of myosin II. J Biol Chem 2004;279:35557-35563. 